\title{
Natural density of relative coprime polynomials in $\mathbb{F}_{q}[x]$
}

Xiangqian Guo, Fengdan Hou, and Xuewen Liu 


\title{
NATURAL DENSITY OF RELATIVE COPRIME POLYNOMIALS IN $\mathbb{F}_{q}[x]$
}

\author{
XIANGQIAN GUO, FENGDAN HOU, AND XUEWEN LIU
}

Received 06 December, 2013

\begin{abstract}
Let $\mathbb{F}_{q}[x]$ be the polynomial ring over the finite field $\mathbb{F}_{q}$ containing $q$ elements. We compute the probability that $n$ polynomials in $\mathbb{F}_{q}[x]$ are $k$-wise relatively coprime, using the concept of natural density. As a special case, we get the probability that $n$ polynomials in $\mathbb{F}_{q}[x]$ are pairwise coprime.
\end{abstract}

2010 Mathematics Subject Classification: 11B05; 11T06; 11C08; 60B15.

Keywords: natural density, $k$-wise relatively coprime, irreducible polynomial, $q$-zeta function

\section{INTRODUCTION AND MAIN RESULTS}

Let $\mathbb{N}$ be the set of all positive integers. Dirichlet [2] first discovered an interesting result that relates the probability that two randomly chosen integers are relative prime to the Riemann's zeta function, and the probability turns out to be

$$
\lim _{N \rightarrow \infty} \frac{\left|\left\{(m, n) \in \mathbb{N}^{2} \mid 1 \leq m, n \leq N, \operatorname{gcd}(m, n)=1\right\}\right|}{N^{2}}=\zeta^{-1}(2)=\frac{6}{\pi^{2}},
$$

where $\operatorname{gcd}(m, n)$ denotes the greatest common divisor of $m$ and $n$, and $\zeta(s)$ is the Riemann's zeta function. This result was generalized to the case of several integers, that is, the probability of $n$ randomly chosen integers to be coprime is given by

$$
\begin{aligned}
\lim _{N \rightarrow \infty} \frac{\left|\left\{\left(m_{1}, \ldots, m_{n}\right) \in \mathbb{N}^{n} \mid 1 \leq m_{1}, \ldots, m_{n} \leq N, \operatorname{gcd}\left(m_{1}, \ldots, m_{n}\right)=1\right\}\right|}{N^{n}} \\
=\zeta^{-1}(n) .
\end{aligned}
$$

In [7], Kubota and Sugita gave a rigorous probabilistic interpretation to Dirichlet's theorem. Other probability problems over integers were also considered: L. Tóth [12] obtained that the probability of $n$ positive integers to be pairwise coprime is $\prod_{p}(1-$ $\left.\frac{1}{p}\right)^{n-1}\left(1+\frac{n-1}{p}\right)$, where $p$ is a prime number; Hu [4] showed that the probability of $n$ positive integers to be $k$-wise relatively prime is $\prod_{p}\left(\sum_{m=0}^{k-1}\left(\begin{array}{c}n \\ m\end{array}\right)\left(\frac{1}{p}\right)^{m}\left(1-\frac{1}{p}\right)^{n-m}\right)$. For deeper links between probability theory and number theory, please refer to Tenenbaum [11], Kubilius [6] and Kac [5]. 
This notation of probability with respect to the uniform distribution over infinite sets $\mathbb{N}^{n}, n \in \mathbb{N}$, is also known as natural density, which can be defined for any subset $A$ as

$$
D(A)=\lim _{N \rightarrow \infty} \frac{\left|A \cap\{1,2, \cdots, N\}^{n}\right|}{N^{n}},
$$

provided the limit exists, where $|\cdot|$ denotes the cardinality of the corresponding set. In [8], Maze, Rosenthal and Wagner computed the natural density of the set of $k \times n$ unimodular integer matrices for any positive integers $k \leq n$, where a $k \times n$ integer matrix is called unimodular if it can be extended to an invertible $n \times n$ matrix over the integers. Recently, Guo and Yang [3] generalized this result to the matrices of polynomials over finite fields.

Let $\mathbb{F}_{q}$ be the finite field consisting of $q$ elements, and $\mathbb{F}_{q}[x]$ be the polynomial ring over $\mathbb{F}_{q}$, where $q$ is a prime power. To define the concept of natural density for certain subsets, we need to enumerate polynomials in $\mathbb{F}_{q}[x]$. For convenience, denote the elements in $\mathbb{F}_{q}$ by $a_{0}=0, a_{1}, \cdots, a_{q-1}$. Let $\Sigma$ be the set of all vectors $\alpha=\left(a_{m_{0}}, a_{m_{1}}, \cdots\right)$ with $m_{i} \in\{0,1, \cdots, q-1\}$ and $m_{i}=0$ for sufficiently large $i$. Then there is a one-to-one map

$$
\chi: \Sigma \rightarrow \mathbb{Z}_{+}=\mathbb{N} \cup\{0\}, \quad \chi\left(a_{m_{0}}, a_{m_{1}}, \cdots\right)=\sum_{j=0}^{\infty} m_{i} q^{i} .
$$

For all $j \in \mathbb{Z}_{+}$, we set

$$
f_{j}(x)=\sum_{i=0}^{\infty} a_{m_{i}} x^{i}, \text { with } \chi\left(a_{m_{0}}, a_{m_{1}}, \cdots\right)=j .
$$

Then $\mathbb{F}_{q}[x]=\left\{f_{j}(x) \mid j \in \mathbb{Z}_{+}\right\}$.

From now on, we fix a prime power $q$ and a positive integer $n \geq 2$. Denote $\mathcal{M}=$ $\left(\mathbb{F}_{q}[x]\right)^{n}$ for convenience and let $\mathcal{M}_{N}$ be the subset of $\mathcal{M}$ consisting of vectors with entries taken from $\left\{f_{0}, f_{1}, \cdots, f_{N}\right\}$. For any subset $S \subseteq \mathcal{M}$, we define the natural density of $S$ in $\mathcal{M}$ as

$$
D(S)=\lim _{N \rightarrow \infty} \frac{\left|S \cap \mathcal{M}_{N}\right|}{\left|\mathcal{M}_{N}\right|} .
$$

Using a probabilistic method, Sugita and Takanobu [10] determined the probability of two polynomials over $\mathbb{F}_{p}$ to be coprime for a prime $p$. Recently, Morrison [9], Benjamin and Bennett [1] computed the probability that $n$ polynomials over $\mathbb{F}_{q}$ are coprime, which is $1-q^{1-n}$. They used natural density methods and Euclidean algorithm respectively. Then it is natural to consider the questions: what is the probability that $n$ polynomials in $\mathbb{F}_{q}[x]$ are pairwise coprime? Generally, what is the probability that $n$ polynomials in $\mathbb{F}_{q}[x]$ are $k$-wise relatively coprime?

Our main purpose in this paper is to compute the probabilities mentioned above. More precisely, we determined the natural density of the set of $n$-dimensional vectors 
over $\mathbb{F}_{q}[x]$ whose entries are $k$-pairwise coprime, for any positive integer $k \leq n$. Our methods are conceptional and the main idea comes from [8] and [3].

Theorem 1. Let $k$ be a positive integer and $k \leq n$. Denote

$$
G=\left\{\left(g_{1}, \cdots, g_{n}\right) \in \mathcal{M} \mid \operatorname{gcd}\left(g_{i_{1}}, \cdots, g_{i_{k}}\right)=1, \forall 1 \leq i_{1}<\cdots<i_{k} \leq n\right\} .
$$

Then the natural density of $G$ is

$$
\prod_{m=1}^{\infty}\left(\sum_{i=0}^{k-1}\left(\begin{array}{l}
n \\
i
\end{array}\right)\left(\frac{1}{q^{m}}\right)^{i}\left(1-\frac{1}{q^{m}}\right)^{n-i}\right)^{\phi(m)},
$$

where $\phi(m)$ is the number of monic irreducible polynomials with degree $m$ in $\mathbb{F}_{q}[x]$.

Remark 1 . The result of Theorem 1 can be understood as follows: the probability that $n$ polynomials in $\mathbb{F}_{q}[x]$ are $k$-wise relatively coprime is

$$
\prod_{m=1}^{\infty}\left(\sum_{i=0}^{k-1}\left(\begin{array}{l}
n \\
i
\end{array}\right)\left(\frac{1}{q^{m}}\right)^{i}\left(1-\frac{1}{q^{m}}\right)^{n-i}\right)^{\phi(m)} .
$$

Take $k=n$, we get that the probability of $n$ polynomials in $\mathbb{F}_{q}[x]$ being coprime is the $\prod_{m=1}^{\infty}\left(1-\frac{1}{q^{m n}}\right)^{\phi(m)}$. To see what this means, we introduce the following $q$-zeta function

$$
\zeta_{q}(n):=\prod_{f}\left(1-\frac{1}{q^{n \operatorname{deg}(f)}}\right)^{-1}=\prod_{m=1}^{\infty}\left(1-\frac{1}{q^{n m}}\right)^{-\phi(m)},
$$

where $f$ goes through all monic irreducible polynomials (not including the constant polynomials, as usual) in $\mathbb{F}_{q}[x]$. Recall the following interesting equation

$$
\prod_{f}\left(1-t^{\operatorname{deg}(f)}\right)^{-1}=\sum_{l=0}^{\infty} q^{l} t^{l}=\frac{1}{1-q t} .
$$

For more details, see [9] and [3]. Putting $t=q^{-n}$ in (1.4), we get

$$
\zeta_{q}^{-1}(n)=1-\frac{1}{q^{n-1}} .
$$

Combining the equations (1.3) and (1.5), we get that the probability of $n$ polynomials in $\mathbb{F}_{q}[x]$ being coprime is $\zeta_{q}^{-1}(n)=1-\frac{1}{q^{n-1}}$, which is just one of the main results of [9]. In particular, when $n=2$, the probability that 2 polynomials in $\mathbb{F}_{q}[x]$ are coprime is $1-\frac{1}{q}$, which is one of the main results of [1].

Taking $k=2$ in Theorem 1, we have the following corollary. 
Corollary 1. Denote $E=\left\{\left(g_{1}, \cdots, g_{n}\right) \in \mathcal{M} \mid \operatorname{gcd}\left(g_{i}, g_{j}\right)=1, \forall 1 \leq i<j \leq n\right\}$. Then

$$
D(E)=\prod_{m=1}^{\infty}\left(\left(1-\frac{1}{q^{m}}\right)^{n-1}\left(1+\frac{n-1}{q^{m}}\right)\right)^{\phi(m)} .
$$

Similarly, the value in (1.6) can be interpreted as the probability that $n$ polynomials in $\mathbb{F}_{q}[x]$ are pairwise coprime.

\section{Results}

In this section, we will give the proof of Theorem 1. Before this, we need some preparations.

Fix a positive integer $k \leq n$. Let $T$ be a finite set of monic irreducible polynomials in $\mathbb{F}_{q}[x]$, denote

$$
\begin{gathered}
G_{T}=\left\{\left(g_{1}, \cdots, g_{n}\right) \in \mathcal{M} \mid f \nmid \operatorname{gcd}\left(g_{i_{1}}, \cdots, g_{i_{k}}\right),\right. \\
\left.\forall f \in T, 1 \leq i_{1}<\cdots<i_{k} \leq n\right\} .
\end{gathered}
$$

Clearly we have $G=\bigcap_{T} G_{T}$. Denote by $\langle f\rangle$ the ideal generated by $f \in \mathbb{F}_{q}[x]$.

Lemma 1. Let $G_{T}$ be defined as above, then we have

$$
D\left(G_{T}\right)=\prod_{f \in T} \sum_{i=0}^{k-1}\left(\begin{array}{l}
n \\
i
\end{array}\right)\left(\frac{1}{q^{\operatorname{deg}(f)}}\right)^{i}\left(1-\frac{1}{q^{\operatorname{deg}(f)}}\right)^{n-i} .
$$

Proof. Denote $f^{(T)}=\prod_{f \in T} f$ and $d_{T}=\operatorname{deg}\left(f^{(T)}\right)$. Given $g \in \mathbb{F}_{q}[x]$ let $\bar{g}$ be its image in $\mathbb{F}_{q}[x] /\left\langle f^{(T)}\right\rangle$. Then for any positive integer $N$, we have the canonical maps

and

$$
\pi: \mathcal{M}_{N} \rightarrow\left(\mathbb{F}_{q}[x] /\left\langle f^{(T)}\right\rangle\right)^{n}, \quad\left(g_{1}, \cdots, g_{n}\right) \mapsto\left(\bar{g}_{1}, \cdots, \bar{g}_{n}\right),
$$

$$
\varphi:\left(\mathbb{F}_{q}[x] /\left\langle f^{(T)}\right\rangle\right)^{n} \rightarrow\left(\prod_{f \in T} \mathbb{F}_{q}[x] /\langle f\rangle\right)^{n} \rightarrow \prod_{f \in T}\left(\mathbb{F}_{q}[x] /\langle f\rangle\right)^{n},
$$

where the first part of $\varphi$ is induced from the isomorphism

$$
\mathbb{F}_{q}[x] /\left\langle f^{(T)}\right\rangle \cong \prod_{f \in T} \mathbb{F}_{q}[x] /\langle f\rangle,
$$

a consequence of the Chinese Remainder Theorem and the second part of $\varphi$ is an obvious isomorphism of vector spaces.

First suppose that $N=m q^{d_{T}}-1$ for some $m \in \mathbb{N}$. Then it is easy to see

$$
\left\{f_{l}(x) \mid 0 \leq l \leq N\right\}=\left\{f_{s}(x) x^{d_{T}}+f_{t}(x) \mid 0 \leq s \leq m-1,0 \leq t \leq q^{d_{T}}-1\right\} .
$$

For any fixed $0 \leq s \leq m-1$, the following projection is one-to-one:

$$
\left\{f_{s}(x) x^{d_{T}}+f_{t}(x) \mid 0 \leq t \leq q^{d_{T}}-1\right\} \longrightarrow \mathbb{F}_{q}[x] /\left\langle f^{(T)}\right\rangle,
$$


and the canonical projection

$$
\left\{f_{l}(x) \mid 0 \leq l \leq N\right\} \longrightarrow \mathbb{F}_{q}[x] /\left\langle f^{(T)}\right\rangle
$$

is $m$-to-one. Thus the projection map $\pi$ is $m^{n}$-to-one.

For any $f \in T$, let $\varphi_{f}$ be the canonical projection from $\left(\mathbb{F}_{q}[x] /\left\langle f^{(T)}\right\rangle\right)^{n}$ to $\left(\mathbb{F}_{q}[x] /\langle f\rangle\right)^{n}$ via $\varphi$. Given any $A \in \mathcal{M}_{N}$, we see that $A \in G_{T}$ if and only if at most $k-1$ entries of $\varphi_{f} \circ \pi(A)$ is zero for all $f \in T$. Noticing that $\left|\mathbb{F}_{q}[x] /\langle f\rangle\right|=q^{\operatorname{deg}(f)}$, it is easy to deduce that

$$
\left|\varphi \circ \pi\left(\mathcal{M}_{N}\right)\right|=\prod_{f \in T} \sum_{i=0}^{k-1}\left(\begin{array}{l}
n \\
i
\end{array}\right)\left(q^{\operatorname{deg}(f)}-1\right)^{n-i} .
$$

As a result we have

$$
\begin{gathered}
\left|G_{T} \bigcap \mathcal{M}_{N}\right|=m^{n}\left|\varphi \circ \pi\left(\mathcal{M}_{N}\right)\right| \\
=\left(m q^{d_{T}}\right)^{n} \prod_{f \in T} \sum_{i=0}^{k-1}\left(\begin{array}{l}
n \\
i
\end{array}\right)\left(\frac{1}{q^{\operatorname{deg}(f)}}\right)^{i}\left(1-\frac{1}{q^{\operatorname{deg}(f)}}\right)^{n-i} .
\end{gathered}
$$

Now let $N$ be any positive integer. There exist $m, r \in \mathbb{Z}_{+}$such that $N+1=$ $m q^{d_{T}}+r$, where $0 \leq r<q^{d_{T}}$ and $m, r$ are not both 0 . For convenience, set $\widetilde{N}=$ $m q^{d_{T}}-1$. Then by the definition of the natural density, we have

$$
\begin{aligned}
D\left(G_{T}\right) & =\lim _{N \rightarrow \infty} \frac{\left|G_{T} \cap \mathcal{M}_{N}\right|}{\left|\mathcal{M}_{N}\right|} \\
& =\lim _{N \rightarrow \infty} \frac{\left|G_{T} \bigcap \mathcal{M}_{\widetilde{N}}\right|+\left|G_{T} \bigcap\left(\mathcal{M}_{N}-\mathcal{M}_{\widetilde{N}}\right)\right|}{\left|\mathcal{M}_{N}\right|} .
\end{aligned}
$$

Note that $\left|\mathcal{M}_{N}-\mathcal{M}_{\widetilde{N}}\right| \leq r n(N+1)^{n-1}$, that is

$$
\lim _{N \rightarrow \infty} \frac{\left|G_{T} \bigcap\left(\mathcal{M}_{N}-\mathcal{M}_{\widetilde{N}}\right)\right|}{\left|\mathcal{M}_{N}\right|} \leq \lim _{N \rightarrow \infty} \frac{r n(N+1)^{n-1}}{(N+1)^{n}}=0 .
$$

So, we obtain

$$
\begin{aligned}
D\left(G_{T}\right) & =\lim _{N \rightarrow \infty} \frac{\left|G_{T} \bigcap \mathcal{M}_{\widetilde{N}}\right|}{(N+1)^{n}} \\
& =\lim _{N \rightarrow \infty} \frac{\left(m q^{d_{T}}\right)^{n} \prod_{f \in T} \sum_{i=0}^{k-1}\left(\begin{array}{c}
n \\
i
\end{array}\right)\left(\frac{1}{q^{\operatorname{deg}(f)}}\right)^{i}\left(1-\frac{1}{q^{\operatorname{deg}(f)}}\right)^{n-i}}{(N+1)^{n}} \\
& =\prod_{f \in T} \sum_{i=0}^{k-1}\left(\begin{array}{l}
n \\
i
\end{array}\right)\left(\frac{1}{q^{\operatorname{deg}(f)}}\right)^{i}\left(1-\frac{1}{q^{\operatorname{deg}(f)}}\right)^{n-i} .
\end{aligned}
$$


This completes the proof.

Proof of Theorem 1.1. For any irreducible polynomial $f \in \mathbb{F}_{q}[x]$, denote

$$
K_{f}=\left\{\left(g_{1}, \cdots, g_{n}\right)|f| \operatorname{gcd}\left(g_{i_{1}}, \cdots, g_{i_{k}}\right), 1 \leq i_{1}<\cdots<i_{k} \leq n\right\} .
$$

Let $q_{f}=q^{\operatorname{deg}(f)}$, then by Lemma 2.1 we have

$$
\begin{aligned}
D\left(K_{f}\right) & =1-D\left(G_{\{f\}}\right) \\
& =1-\sum_{i=0}^{k-1}\left(\begin{array}{l}
n \\
i
\end{array}\right)\left(\frac{1}{q_{f}}\right)^{i}\left(1-\frac{1}{q_{f}}\right)^{n} \\
& \leq 1-\left(1-\frac{n-1}{q_{f}}\right)\left(1+\frac{n-1}{q_{f}}\right) \\
& =\left(\frac{n-1}{q_{f}}\right)^{2} .
\end{aligned}
$$

Let $T_{t}$ be the set of all monic irreducible polynomials with degree no more than $t$, and denote $\widehat{T}$ the set of all monic irreducible polynomials in $\mathbb{F}_{q}[x]$. For convenience, we set $G_{t}=G_{T_{t}}$. Since

$$
\left(G_{t} \backslash G\right) \subseteq \bigcup_{f \in \hat{T} \backslash T_{t}} K_{f}
$$

we have

$$
\begin{aligned}
\limsup _{N \rightarrow \infty} \frac{\left|\left(G_{t} \backslash G\right) \cap \mathcal{M}_{N}\right|}{\left|\mathcal{M}_{N}\right|} & \leq \limsup _{N \rightarrow \infty} \frac{\left|\left(\bigcup_{f \in \hat{T} \backslash T_{t}} K_{f}\right) \cap \mathcal{M}_{N}\right|}{\left|\mathcal{M}_{N}\right|} \\
& \leq \limsup _{N \rightarrow \infty} \frac{\sum_{f \in \hat{T} \backslash T_{t}}\left|K_{f} \bigcap \mathcal{M}_{N}\right|}{\left|\mathcal{M}_{N}\right|} \\
& \leq \sum_{f \in \hat{T} \backslash T_{t}} \limsup _{N \rightarrow \infty} \frac{\left|K_{f} \bigcap \mathcal{M}_{N}\right|}{\left|\mathcal{M}_{N}\right|} \\
& =\sum_{f \in \hat{T} \backslash T_{t}} D\left(K_{f}\right)<\sum_{f \in \hat{T} \backslash T_{t}}\left(\frac{n-1}{q_{f}}\right)^{2} \\
& =\sum_{m=t+1}^{\infty} \frac{(n-1)^{2}}{q^{2 m}} \phi(m),
\end{aligned}
$$

where $\phi(m)$ denotes the number of monic irreducible polynomials with degree $m$ in $\mathbb{F}_{q}[x]$. 
Since all irreducible polynomials with degree $m$ can divide $x^{q^{m}}-x$, which has no multiple roots, thus $m \phi(m) \leq q^{m}$ and

$$
\limsup _{N \rightarrow \infty} \frac{\left|\left(G_{t} \backslash G\right) \bigcap \mathcal{M}_{N}\right|}{\left|\mathcal{M}_{N}\right|} \leq \sum_{m=t+1}^{\infty} \frac{(n-1)^{2}}{m q^{m}} \leq \frac{(n-1)^{2}}{q^{t}(q-1)} .
$$

Note that $G \bigcap \mathcal{M}_{N} \subseteq G_{t} \bigcap \mathcal{M}_{N}$ and $G \bigcap \mathcal{M}_{N}=G_{t} \cap \mathcal{M}_{N}-\left(G_{t} \backslash G\right) \bigcap \mathcal{M}_{N}$, which imply that

$$
\limsup _{N \rightarrow \infty} \frac{\left|G \bigcap \mathcal{M}_{N}\right|}{\left|\mathcal{M}_{N}\right|} \leq \limsup _{N \rightarrow \infty} \frac{\left|G_{t} \bigcap \mathcal{M}_{N}\right|}{\left|\mathcal{M}_{N}\right|} \leq D\left(G_{t}\right)
$$

and

$$
\begin{aligned}
\liminf _{N \rightarrow \infty} \frac{\left|G \bigcap \mathcal{M}_{N}\right|}{\left|\mathcal{M}_{N}\right|} & \geq \liminf _{N \rightarrow \infty} \frac{\left|G_{t} \bigcap \mathcal{M}_{N}\right|}{\left|\mathcal{M}_{N}\right|}-\limsup _{N \rightarrow \infty} \frac{\left(G_{t} \backslash G\right) \bigcap \mathcal{M}_{N}}{\left|\mathcal{M}_{N}\right|} \\
& \geq D\left(G_{t}\right)-\frac{(n-1)^{2}}{q^{t}(q-1)},
\end{aligned}
$$

for all $t \in \mathbb{N}$. Let $t$ tend to $\infty$, from Lemma 2.1, we can conclude that

$$
\begin{aligned}
\lim _{N \rightarrow \infty} \frac{\left|G \bigcap \mathcal{M}_{N}\right|}{\left|\mathcal{M}_{N}\right|} & =\lim _{t \rightarrow \infty} D\left(G_{t}\right) \\
& =\lim _{t \rightarrow \infty} \prod_{f \in T_{t}} \sum_{i=0}^{k-1}\left(\begin{array}{l}
n \\
i
\end{array}\right)\left(\frac{1}{q_{f}}\right)^{i}\left(1-\frac{1}{q_{f}}\right)^{n-i} \\
& =\lim _{t \rightarrow \infty} \prod_{m=1}^{t} \sum_{i=0}^{k-1}\left(\begin{array}{l}
n \\
i
\end{array}\right)\left(\frac{1}{q^{m}}\right)^{i}\left(1-\frac{1}{q^{m}}\right)^{n-i} \\
& =\prod_{m=1}^{\infty}\left(\sum_{i=0}^{k-1}\left(\begin{array}{l}
n \\
i
\end{array}\right)\left(\frac{1}{q^{m}}\right)^{i}\left(1-\frac{1}{q^{m}}\right)^{n-i}\right)^{\phi(m)} .
\end{aligned}
$$

This completes the proof.

\section{ACKNOWLEDGEMENT}

This work is partially supported by the NSF of China (Grant No. 11101380, 11471294). The authors would like to express their gratitude to the referees for valuable suggestions.

\section{REFERENCES}

[1] A. T. Benjamin and C. D. Bennett, "The probability of relatively prime polynomials," Math. Mag., vol. 80, pp. 196-202, 2007. 
[2] G. L. Dirichlet, Über die Bestimmung der mittleren Werthe in der Zahlentheorie. Abhandlungen Königlich Preuss, Akad. Wiss., 1849.

[3] X. Guo and G. Yang, "The probability of rectangular unimodular matrices over $\mathbb{F}_{q}[x]$," Linear Algebra Appl., vol. 438, pp. 2657-2682, 2013.

[4] J. Hu, "The probability that random positive integers are $k$-wise relatively prime," Int. J. Number Theory, vol. 09, 2013.

[5] M. Kac, Statistical independence in probability, analysis and number theory, ser. The Carus Mathematical Monographs. New York: Mathematical Association of America, John Wiley and Sons, Inc., 1959, vol. 16.

[6] J. Kubilius, "Probabilistic methods in the theory of numbers," Amer. Math. Soc. Transl. (2), vol. 19, pp. 47-85, 1962

[7] H. Kubota and H. Sugita, "Probabilistic proof of limit theorems in number theory by means of adeles," Kyushu J. Math., vol. 56, pp. 391-404, 2002.

[8] G. Maze, J. Rosenthal, and U. Wagner, "Natural density of rectangular unimodular integer matrices," Linear Algebra Appl., vol. 434, pp. 1319-1324, 2011.

[9] K. E. Morrison, "Random polynomials over finite fields," http://www.calpoly.edu/ kmorriso/Research/RPFF.pdf, 1999.

[10] H. Sugita and S. Takanobu, "The probability of two $\mathbb{F}_{q}$-polynomials to be coprime," Adv. Stud. Pure Math., vol. 49, pp. 455-478, 2007.

[11] G. Tenenbaum, Introduction to analytic and probabilistic number theory, ser. Cambridge Studies in Advanced Mathematics. Cambridge: Cambridge University Press, 1995, vol. 46.

[12] L. Tóth, "The probability that $k$ positive integers are pairwise relatively prime," Fibonacci Quart., vol. 40, pp. 13-18, 2002.

Authors' addresses

Xiangqian Guo

Zhengzhou University, School of Mathematics and Statistics, 100 Science Road, 450001 Zhengzhou, P. R. China

E-mail address: guoxq@ZZu.edu.cn

Fengdan Hou

Zhengzhou University, School of Mathematics and Statistics, 100 Science Road, 450001 Zhengzhou, P. R. China

E-mail address: houfdezzu.ps.edu.cn

Xuewen Liu

Zhengzhou University, School of Mathematics and Statistics, 100 Science Road, 450001 Zhengzhou, P. R. China

E-mail address: liuxw@zzu.edu.cn 